\title{
Non-Adiabatic Effects in the Time Dependent Level Crossing Problem*
}

\author{
A. Góźdź ${ }^{1}$, E. Werner, and M. Brack \\ Institut für Theoretische Physik, Universität Regensburg, \\ Federal Republic of Germany
}

Received May 23, 1984

\begin{abstract}
We discuss the exact solution of the time-dependent Schrödinger equation for a system of two crossing levels with a residual interaction. In contrast to the familiar LandauZener (LZ) solution used in most applications, we allow for more general boundary conditions; in particular we treat explicitly the case of a finite interval around the crossing point. The exact jumping probability is shown to be extremely sensitive to these boundary conditions; in many realistic cases it is found to be smaller than the LZ value by several orders of magnitude. We also compare the exact excitation energy to the one obtained in the usual cranking approach.
\end{abstract}

\section{Introduction}

Most microscopic models for nuclear collective motion use a deformed single-particle hamiltonian to create a single particle basis dependent on a set of collective variables along which the collective motion is supposed to take place. For any two single particle states treated as functions of collective variables the phenomenon of level crossing can occur. The main part of nonadiabatic effects of a physical system is often supposed to be due to such intersections of energy levels; then different kinds of "hopping models" can be applied to describe corrections to adiabatic collective motion [1-4]. In applications, the evaluation of the jump probability at level crossings is mostly based on the known Landau-Zener solution for a two-level system [5-6]. However, this particular solution is obtained with somewhat unrealistic conditions: the initial and final deformations have to be very different from the deformation at the crossing point while the collective velocity is assumed to be constant. In addition, as it is supposed in hopping models, the two-level approximation is reasonable only as a local approximation in the vicinity of the crossing point for two single-particle levels. Therefore a better estimation of

\footnotetext{
* Work supported in parts by GSI Darmstadt

1 Permanent address: Department of Physics, The University of Maria Sklodowska-Curie, Pl-20-031 Lublin, Poland
}

the jump probability than given by the LandauZener formula is needed. In the literature, the full treatment of the Landau-Zener model is usually restricted to applications in atomic and molecular physics with their specific conditions dictated by the physics of the problem [7-9]. The aim of the present paper is an analysis of the LZ-model from the point of view of its application to nuclear large amplitude collective motion. For this purpose, in Sect. 2 the full analytical solution of the Landau-Zener model is quoted [10-12] with a suitable notation and for arbitrary initial conditions. The general formulae for transition probabilities between pairs of diabatic or adiabatic states are derived in Sect. 3. The discussion in Sect. 4 shows that the transition probability is a function not only of a diabacity parameter $[13,14$, $3,4]$ but also of a deformation-dependent complex variable; its behaviour is more complicated than the LZ-formula suggests $[1,3,4,14]$, as soon as more realistic, finite deformation intervals are considered.

In the past, a lot of activities were devoted to different approximations and extensions of the LZmodel. A revue of these attempts can be found in the papers $[7-11,16]$ and in the references therein. It seems that our approach to the LZ-model is slightly similar to that proposed by Heinrichs [11]. 
However, his analysis is restricted to special boundary conditions which are usually not fulfilled in largeamplitude collective nuclear motion. In addition his resulting transition probability is not a nonnegative function because of approximations which were involved in its derivation.

In Sect. 5 we compare the exact excitation energy of the two-level system with the excitation energy calculated from the cranking formula. Large relative deviations from the cranking estimate are observed not only for typical collective velocities occuring e.g. in the fission process, but also for relatively slow collective motion, with $\dot{\alpha} \sim$ a hundred times smaller. The magnitudes of these deviations are, clearly, not too large for small $\dot{\alpha}$. However, if a great number of crossing points is involved in large-amplitude nuclear collective motion, they can have a great effect giving a significant contribution to the excitation energy due to non-adiabacity of the motion. It is also important to note that the independence of transitions at successive crossing points, being one of the basic assumptions of different "hopping models", is often violated (see Sect. 4).

\section{The Two-Level Crossing Problem}

The most general single-particle hamiltonian for a two-level fermion system can be written in terms of a fictitious $1 / 2$ spin

$H=\sum_{m, m^{\prime}} \varepsilon_{m m^{\prime}} a_{m}^{+} a_{m^{\prime}}=\varepsilon_{s}+\gamma \hat{\tau}_{0}+\delta\left(e^{-i \varphi} \hat{\tau}_{+}+e^{i \varphi} \hat{\tau}_{-}\right)$,

where $\varepsilon_{s}=1 / 2 \sum_{m} \varepsilon_{m m}$ represents the average energy of the levels (in further considerations we put it equal to zero because it affects only a shift of the energy spectrum); $\gamma=\sum_{m} \operatorname{sgn}(m) \varepsilon_{m m}$ stands for the energy difference of the levels, and a coupling between the levels is described by

$\delta e^{-i \varphi}=\varepsilon_{\frac{1}{2}-\frac{1}{2}}=\left(\varepsilon_{-\frac{1}{2} \frac{1}{2}}\right)^{*} \quad$ with $\delta \geqq 0$ and $\varphi \in[0,2 \pi[$.

The operators $a_{m}^{+}$and $a_{m}$ are fermion creation and annihilation operators, respectively. $\hat{\tau}_{0}, \hat{\tau}_{+}$and $\hat{\tau}_{-}$ are defined in the form

$\hat{\tau}_{0}=\frac{1}{2}\left(a_{\frac{1}{2}}^{+} a_{\frac{1}{2}}-a_{-\frac{1}{2}}^{+} a_{-\frac{1}{2}}\right) \quad \hat{\tau}_{+}=\left(\hat{\tau}_{-}\right)^{+}=a_{\frac{1}{2}}^{+} a_{-\frac{1}{2}}$

and stand for the generators of the fictitious $S U(2)$ spin. Using the fictitious-spin formalism we want to underline the transformation properties of the twolevel system; all possible continuous unitary transformations can be understood as rotations in the fictitious-spin space. We suppose the hamiltonian (1) to be a linear function of only one collective variable $\alpha$,
$[5,6]$ whereby

$\gamma(\alpha)=b \alpha ; \quad b=$ const

and the coupling terms $\delta$ and $\phi$ are independent of the deformation parameter $\alpha$. In addition we assume uniform collective motion with constant velocity

$\alpha=\dot{\alpha} t=\frac{1}{\hbar} u t, \quad u=$ const $\neq \neq 0$.

This is a rather weak condition since the overall process can always be divided into small intervals between subsequent crossings. The above conditions then have to be satisfied only piecewise in each of the subsequent intervals. The physical meaning of $b$ is the difference of the slopes of the two intersecting levels. With these assumptions we can solve the Schrödinger equation for the system $(\hbar=1)$

$i u \frac{\partial}{\partial \alpha}|\psi(\alpha)\rangle=H(\alpha)|\psi(\alpha)\rangle$

where the total wave function

$|\psi(\alpha)\rangle=c_{\uparrow}(\alpha)\left|\frac{1}{2}\right\rangle+c_{\downarrow}(\alpha)\left|-\frac{1}{2}\right\rangle$

and

$\left| \pm \frac{1}{2}\right\rangle \equiv a_{ \pm \frac{1}{2}}^{+}|0\rangle$

define a deformation-independent basis. The general solution of (5) with the initial amplitudes at a deformation $\alpha_{0}$ denoted by $c_{\uparrow}^{0}$ and $c_{\downarrow}^{0}$ can be written in the form

$\mathcal{c}_{\uparrow}\left(G ; x_{0}, x\right)=K^{-1}\left(G_{0} ; x_{0}, x_{0}\right)$

. $\left\{c_{\uparrow}^{0} K\left(G_{0} ; x_{0}, x\right)+c_{\downarrow}^{0} A\left(G ; x_{0}, x\right)\right\}$,

$c_{\downarrow}\left(G ; x_{0}, x\right)=K^{-1}\left(G_{0} ; x_{0}, x_{0}\right)$

$\cdot\left\{-c_{\uparrow}^{0} A^{*}\left(G ; x_{0}, x\right)+c_{\downarrow}^{0} K^{*}\left(G_{0} ; x_{0}, x\right)\right\}$

where

$K\left(G_{0} ; x_{0}, x\right)=\sqrt{2}\left[\left(E_{\mu}^{(0)}\left(x_{0}\right)\right)^{*} E_{\mu}^{(0)}(x)\right.$

$\left.+\frac{1}{4 \pi G_{0}}\left(E_{\mu}^{(1)}\left(x_{0}\right)\right)^{*} E_{\mu}^{(1)}(x)\right]$,

$A\left(G ; x_{0}, x\right)=\frac{e^{-i \frac{\pi}{4}}}{\sqrt{2 \pi} G^{*}}\left[E_{\mu}^{(0)}\left(x_{0}\right) E_{\mu}^{(1)}(x)\right.$

$\left.-E_{\mu}^{(1)}\left(x_{0}\right) E_{\mu}^{(0)}(x)\right]$.

The complex diabacity parameter $G$ is defined by (bu>0)

$G=e^{-i \varphi} \sqrt{\frac{b u}{2 \pi \delta^{2}}}=\sqrt{G_{0}} e^{-i \varphi}$. 
Furthermore we have defined

$\mu=\frac{i}{2 \pi G_{0}}-1$ and $x_{0}=e^{-i \frac{\pi}{4}} \sqrt{\frac{b}{u}} \alpha_{0}$.

The functions $E_{v}^{(0)}$ and $E_{v}^{(1)}$ are combinations of the standard parabolic cylinder functions $[12,15]$ :

$$
\begin{gathered}
E_{v}^{(0)}(z)=\frac{\Gamma\left(\frac{1-v}{2}\right)}{\sqrt{\pi}} 2^{-\frac{1+v}{2}}\left(D_{v}(z)+D_{v}(-z)\right), \\
E_{v}^{(1)}(z)=-\frac{\Gamma\left(-\frac{v}{2}\right)}{\sqrt{\pi}} 2^{-\frac{1+v}{2}}\left(D_{v}(z)-D_{v}(-z)\right) .
\end{gathered}
$$

Note that the amplitudes $c_{\uparrow}$ and $c_{\downarrow}$ are functions of $b, \delta, u, \alpha$ and $\alpha_{0}$ only through the combinations $G, x_{0}$ and $x$. To complete the solution of (5), we quote the result for the limiting case of two parallel energy levels $(b=0)$ coupled by a nonzero nondiagonal term, $\delta>0$ :

$$
\begin{aligned}
& c_{\uparrow}\left(\omega ; \alpha_{0}, \alpha\right)=c_{\uparrow}^{0} \cos \left[\omega\left(\alpha-\alpha_{0}\right)\right] \\
& +c_{\downarrow}^{0} e^{-i\left(\varphi+\frac{\pi}{2}\right)} \sin \left[\omega\left(\alpha-\alpha_{0}\right)\right], \\
& c_{\downarrow}\left(\omega ; \alpha_{0}, \alpha\right)=c_{\downarrow}^{0} \cos \left[\omega\left(\alpha-\alpha_{0}\right)\right] \\
& +c_{\uparrow}^{0} e^{i\left(\varphi-\frac{\pi}{2}\right)} \sin \left[\omega\left(\alpha-\alpha_{0}\right)\right]
\end{aligned}
$$

where

$$
\omega=\left|\frac{\delta}{u}\right|
$$

is a typical spatial frequency of quantum beats in the system.

\section{The Transition Probability}

The probability of a transition having taken place between two states at a deformation $\alpha>0$ is defined as the occupation probability of the upper level calculated with the condition that for the initial deformation $\alpha_{0} \leqq 0$ (before the crossing point) only the lower level is occupied. For example the transition probability between the diabatic states (7) can be evidently written

$J\left(G ; x_{0}, x\right)=\left|c_{\uparrow}\left(G ; x_{0}, x\right)\right|^{2}$,

where $c_{\uparrow}\left(G, x_{0}, x\right)$ is calculated from (11) with the initial conditions $\left|c_{\uparrow}^{0}\right|=1$ and $\left|c_{\downarrow}^{0}\right|=0$ for a fixed value of the collective variable. A little algebra gives the explicit form for the expression (13):
$J\left(G_{0} ; x_{0}, x\right)=\left|\frac{K\left(G_{0} ; x_{0}, x\right)}{K\left(G_{0} ; x_{0}, x_{0}\right)}\right|^{2}$.

Applying the transformation properties of the amplitudes $c_{\uparrow}$ and $c_{\downarrow}$, which are the components of a spherical tensor of rank $1 / 2$ and transform according to the two-dimensional irreducible representation $D^{(1 / 2)}$ of $S U(2)$, we can easily find an expression for the transition probability between the adiabatic states (eigenstates $| \pm\rangle$ ) of this two-level system:

$J_{A}\left(G ; x_{0}, x\right)=\left|c_{+}\left(G ; x_{0}, x\right)\right|^{2}$

$=\frac{1}{2}-\sqrt{\frac{\kappa}{1+\kappa}}\left(\frac{1}{2}-\left|c_{\uparrow}\left(G ; x_{0}, x\right)\right|^{2}\right)$

$+\frac{1}{\sqrt{1+\kappa}} \operatorname{Re}\left\{c_{\uparrow}^{*}\left(G ; x_{0}, x\right) c_{\downarrow}\left(G ; x_{0}, x\right) e^{-i \varphi}\right\}$,

where

$\kappa=\frac{\pi}{2}|x|^{2} G_{0}$.

The initial diabatic amplitudes are given by the equations

$c_{\downarrow}^{0}=\cos \frac{\theta_{0}}{2} \quad$ and $\quad c_{\uparrow}^{0}=-e^{-i \varphi} \sin \frac{\theta_{0}}{2}$.

Here $\phi$ is defined by the hamiltonian (1) and $\theta$ is the angle of rotation between dia- and adiabatic bases:

$\sin \theta=[1+\kappa]^{-\frac{1}{2}}$,

$\cos \theta=\operatorname{sgn}(\operatorname{Re}(x))\left[\frac{\kappa}{1+\kappa}\right]^{\frac{1}{2}}$,

where

$0 \leqq \theta \leqq \pi$.

The value $\theta=0$ is related to $\gamma=+\infty$ and $\theta=\pi$ corresponds to $\gamma=-\infty$.

A typical behaviour of the transition probabilities $J$ and $J_{A}$ as functions of the parameter $x$ for a fixed $x_{0}$ and $G$ is shown in the Fig. 1. This example demonstrates how the transition probability is strongly related to the basis which one uses for calculations. For instance, in the DDD-approach $[3,4,14]$ it must be carefully distinguished if the adiabatic or the diabatic basis is used. Note that in Fig. $1, J$ and $J_{A}$ are plotted in two different scales.

\section{A Geometrical Interpretation of the Two-Intersecting-Levels System}

In Sect. 2, the hamiltonian of the two-level system has been introduced in terms of fictitious spin-1/2 


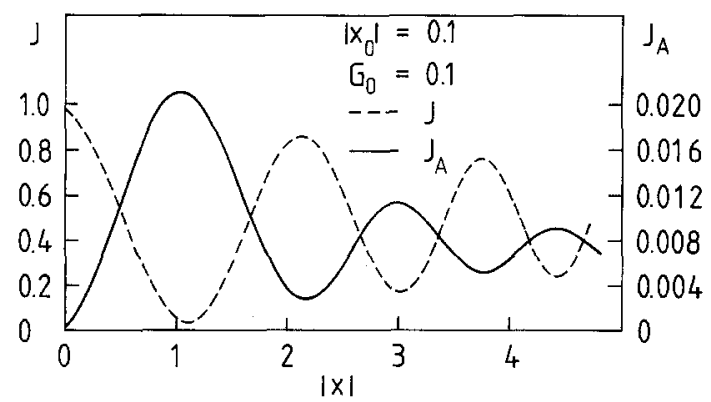

Fig. 1. Typical behaviour of the diabatic (dashed line) and adiabatic (solid line) transition probabilities, in the vicinity of crossing point, as functions of the "deformation" parameter $|x|\left(\left|x_{0}\right|=0.1\right.$ and $G_{0}=0.1$ ). Note, that the figure is plotted in two different scales

operators. The parameters of the hamiltonian can also be interpreted as three components of a fictitious magnetic field

$\mathbf{Q}=2 \delta \cos \varphi \mathbf{e}_{x}+2 \delta \sin \varphi \mathbf{e}_{y}+\dot{\gamma} t \mathbf{e}_{z}$,

where $\mathbf{e}_{x, y, z}$ are the unit cartesian vectors, and $\dot{\gamma}$ $=b u=$ const. The field $\mathbf{Q}$ corresponds to the realistic magnetic field $\mathbf{B}$ by the obvious relation $\mathbf{Q}=-g \mathbf{B}$, where $g$ is the gyromagnetic ratio. The hamiltonian (1) can now be written as the scalar product of the Q-field and the spin vector operator

$H=\mathbf{Q} \cdot \tau, \quad\left(\varepsilon_{s}=0\right)$.

The most general form of the density operator for the spin $1 / 2$ is well known and can be written

$\rho=\frac{1}{2} \mathbb{l}+\mathbf{P} \cdot \tau$.

In this expression the identity operator is denoted by $\mathbb{1}$ and the polarization vector $\mathbf{P}$, which may be said to point in the direction of the spin, is defined by its components

$P_{x}=2 \operatorname{Re}\left(c_{\uparrow}^{*} c_{\downarrow}\right)$,

$P_{y}=2 \operatorname{Jm}\left(c_{\uparrow}^{*} c_{\downarrow}\right)$,

$P_{z}=\left|c_{\uparrow}\right|^{2}-\left|c_{\downarrow}\right|^{2}$.

The vector $P$ satisfies the following equation of motion [17]:

$\hbar \frac{d \mathbf{P}}{d t}=\mathbf{Q} \times \mathbf{P}$.

The system in question is completely described by the magnetic field and the polarization vector. For instance, the total energy and its time derivative are given by two simple equations

$\varepsilon=\frac{1}{2} \mathbf{P} \cdot \mathbf{Q}$

and $\frac{d \varepsilon}{d t}=\frac{1}{2} \mathbf{P} \cdot \frac{d \mathbf{Q}}{d t}=\frac{\dot{\gamma}}{2 \hbar} P_{z}$

The occupation probabilities of the diabatic states are closely related to the $z$-component of the polarization vector

$\left|c_{\uparrow}\right|^{2}=\operatorname{Tr}\left(\left|\frac{1}{2}\right\rangle\left\langle\frac{1}{2}\right| \hat{\rho}\right)=\frac{1}{2}\left(1+P_{z}\right)$

and

$\left|\mathcal{c}_{\downarrow}\right|^{2}=\frac{1}{2}\left(1-P_{z}\right)$.

For the occupation probabilities of the eigenstates we get, respectively,

$\left|\mathcal{c}_{+}\right|^{2}=\frac{1}{2}\left(1+\frac{\mathbf{P} \cdot \mathbf{Q}}{|\mathbf{Q}|}\right)$,

and

$\left|c_{-}\right|^{2}=\frac{1}{2}\left(1-\frac{\mathbf{P} \cdot \mathbf{Q}}{|\mathbf{Q}|}\right)$.

In this geometrical picture, the angles $\theta$ and $\phi$ introduced by the Eqs. (1) and (17) are simply the polar angles of the direction of the field $\mathbf{Q}$ and are determined by the equations

$\theta(t)=\operatorname{arctg}\left(\frac{Q_{z}}{\sqrt{Q_{x}^{2}+Q_{y}^{2}}}\right), \quad 0 \leqq \theta \leqq \pi$

and

$Q_{x}+i Q_{y}=\sqrt{Q_{x}^{2}+Q_{y}^{2}} e^{i \varphi}, \quad 0 \leqq \varphi \leqq 2 \pi$.

Assume now that for an initial value of time $t_{0}$ the polarization vector points in the positive direction of the $z$-axis. It corresponds to the initial conditions needed for calculation of the transition probability between two diabatic states (7). Then the probability of a jump is related to the projection of the polarization vector according to (24).

If the field $\mathbf{Q}$ is changing from its initial value to the final one very quickly, the vector $\mathbf{P}$ practically is still at the same position during the whole process, i.e. the particle stays on the diabatic level for very fast collective motion. For a slowly varying magnetic field, the polarization vector can follow the field, but because of the finite angle between $\mathbf{P}$ and $\mathbf{Q}$ a Larmor-like precession is observed giving strong oscillations of the transition probability in a narrow region near the $x y$ plane, e.g. at the vicinity of the crossing point. The Landau-Zener limit corresponds to taking the initial and final times equal to $-\infty$ and $+\infty$, respectively. In this case no oscillation can be found because all oscillations are strongly damped for $t \rightarrow \pm \infty$. The same analysis can be made using (25) for the transition probability between ei- 


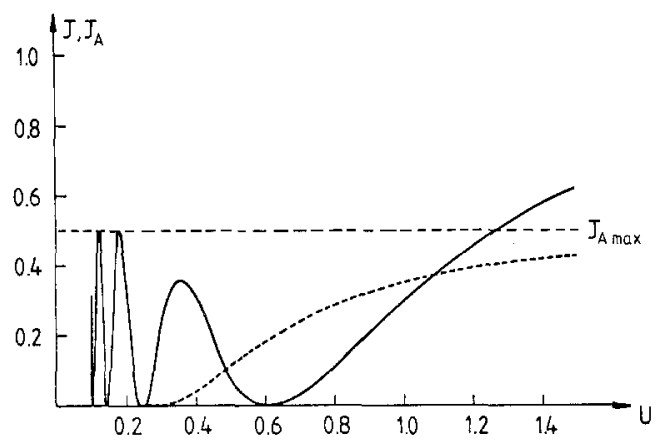

Fig. 2. Typical behaviour of the diabatic (solid line) and adiabatic (dashed line) transition probabilities as functions of the collective velocity $u\left(-\alpha_{0}=\alpha_{0}=1 \mathrm{fm}, b=1 \mathrm{MeV} \cdot \mathrm{fm}^{-1}, \delta=0.5 \mathrm{MeV}, u=h \cdot \alpha\right.$ $\mathrm{MeV} \cdot \mathrm{fm})$

genstates. The initial position of the polarization vector is, however, different from that for the diabatic states. Initially the magnetic field $\mathbf{Q}$ and the polarization vector $\mathbf{P}$ are acutally parallel vectors. For an extremely slowly varying magnetic field $\mathbf{Q}$ the polarization vector $\mathbf{P}$ follows the field and is practically parallel to it. It corresponds to the case when a particle moves along the lower eigenstate the adiabatic case. For a finite velocity the description is more complicated because of the Larmor-like precession. It is always present and leads to large amplitude oscillations in the diabatic transition probability.

In the adiabatic case, with parameters appropriate for fission reactions of heavy nuclei, these oscillations are relatively small. However, we would like to emphasize once more that these two cases cannot be compared as far as a small neighbourhood of the crossing point is concerned. They correspond to completely different initial conditions. The oscillatory behaviour of the diabatic transition probability can be studied by making use of the exact solutions (8) of the Schrödinger equation (5). In Fig. 2, both adiabatic and diabatic transition probabilities are plotted as functions of the collective velocity $u$. The remaining parameters are fixed. Three characteristic features are observed:

i) The frequency of the diabatic transition probability is a strongly decreasing function of the collective velocity.

ii) There exists a specific value of the collective velocity, $u_{c}$, which corresponds to the last minimum of the diabatic transition probability. If the collective velocity is higher than $u_{c}$, the probability of a jump becomes a monotonously increasing function of $u$.

iii) The adiabatic transition probability, $J_{A}$, is bounded by the number $\frac{1}{2}\left\{1-\cos \left(\theta-\theta_{0}\right)\right\} \leqq 1$, when $u \rightarrow \infty$.

In Table 1 the maximal value (i.e. for infinite col-
Table 1. Limiting values (for infinite collective velocity $u$ ) of the adiabatic transition probabilities for the symmetric case, $\left|\alpha_{0}\right|=\alpha$ $=\Delta \alpha$

\begin{tabular}{llll}
\hline$\Delta \alpha[\mathrm{fm}]$ & $b\left[\frac{\mathrm{MeV}}{\mathrm{fm}}\right]$ & $\delta[\mathrm{MeV}]$ & $J_{A \max }=\frac{\kappa}{1+\kappa}$ \\
\hline 0.25 & 1 & 0.25 & 0.2 \\
1 & 1 & 0.25 & 0.8 \\
0.25 & 1 & 0.5 & 0.06 \\
2 & 1 & 0.5 & 0.8 \\
0.25 & 1 & 0.75 & 0.03 \\
2 & 1 & 0.75 & 0.64 \\
0.25 & 3 & 0.5 & 0.36 \\
0.75 & 3 & 0.5 & 0.83 \\
\hline
\end{tabular}

lective velocity) of the adiabatic transition probability is given as a function of distance from the crossing point. This "saturation" property is in contradiction with the analysis of different dynamical nuclear models as a function of the diabacity parameter and the time of local equilibration only $[3,4]$.

The application of the Landau-Zener infinite interval approximation for the case of successive crossings is therefore dangerous. The above saturation property of the adiabatic jumping probability in a finite interval shows that there can be considerable contributions to the transition probability at large distances from the crossing point. Therefore, if the average length of intervals between successive crossings is such that $J_{A}$ is appreciably smaller than unity in one interval, there must be a strong dynamical influence of the "tail" of the foregoing crossings on what happens at a particular one. This means, of course, that there is a strong interdependence of transitions at successive crossings. Consequently, the problem can not be reduced to a series of independent crossings where the situation at the end of the $n$-th inverval serves only to define the initial conditions for the $(n+1)$-th interval.

\section{Adiabatic Cranking and the Excitation Energy}

In the light of the picture obtained in the previous sections a comment on the adiabatic cranking model [18] widely used in nuclear physics is in place. In this section we compare the total excitation energy of the two-level system given by the exact formula (27) and that calculated in a conventional way with the Inglis mass parameter (28). The total excitation energy is obviously related to the adiabatic transition probability $J_{A}$. For given initial and final deformations $\alpha_{0}$ and $\alpha$, respectively, and for arbitrary parameters $b, \delta$ and $u$, the excitation energy can be expressed as 
$E^{*}=\sqrt{b^{2} \alpha^{2}+4 \delta^{2}} J_{A}\left(G ; x_{0}, x\right)$.

Because the transition probability $J_{A}$ depends not only on the actual deformation $\alpha$ but also on the initial one $\alpha_{0}$, the excitation energy depends on the "history" of the particle motion. On the other hand, the appropriate mass parameter calculated from the Inglis formula is given by the simple expression

$B(\alpha, b, \delta)=2 \hbar^{2} \frac{b^{2} \delta^{2}}{\left[b^{2} \alpha^{2}+4 \delta^{2}\right]^{5 / 2}}$,

where no dependence on the initial state is contained. In addition, for a fixed difference of slopes $b$ the mass parameter (28) treated as a function of the deformation $\alpha$ and of the coupling parameter $\delta$ (which corresponds to a linearized or diagonalized residual interaction) exhibits a discontinuity at the point $\alpha=\delta=0$. To see that this behaviour is unphysical it is enough to calculate two limits:

$\lim _{\delta \rightarrow 0} \lim _{\alpha \rightarrow 0} B(\alpha, b, \delta)=\infty$,

$\lim \lim B(\alpha, b, \delta)=0$.

The former indicates that at the crossing point a very small interaction $\delta$ produces a large spike in the mass parameter, the latter shows that if the interaction $\delta$ is being switched off first, the Inglis mass parameter at the crossing point becomes negligible. It is obvious that this strange feature is an unphysical effect caused by the adiabatic approximation [10]. The importance of non-adiabatic effects due to the possibility of a jump of a particle between two single-particle levels is exhibited in Fig. 3. There the excitation energy $E^{*}$ and $E_{\mathrm{c}}=\frac{1}{2} B \dot{\alpha}^{2}$ are plotted as functions of the deformation parameter $\alpha$. The typical collective velocity in a fission process between the saddle and scission point is of the order $10^{20}-10^{21} \frac{\mathrm{fm}}{\mathrm{s}}$ (corresponding to $u \sim 0.1-1 \mathrm{Me} \cdot \mathrm{fm}$ ). It is seen from Fig. 3 that in this range of collective velocities the behaviour of $E^{*}$ and $E_{c}$ differs even qualitatively. For much smaller collective velocity, $u \sim 0.01, E^{*}$ becomes an oscillating function behaving approximately as a combination of $\sin ^{2} \omega \alpha$ and $\cos ^{2} \omega \alpha$ functions, where $\omega$ is the frequency of quantum beats (12a), as is shown in Fig. 3b. Even in this case of very small collective velocity the average of $E^{*}$ is nearly 4 times larger than the cranking model prediction. The same features are seen in Figs. 3c and $3 \mathrm{~d}$ where a different slope parameter has been used. From the results one can see that the adiabatic cranking formula describes only a part of the total excitation energy for very slow collective motion,

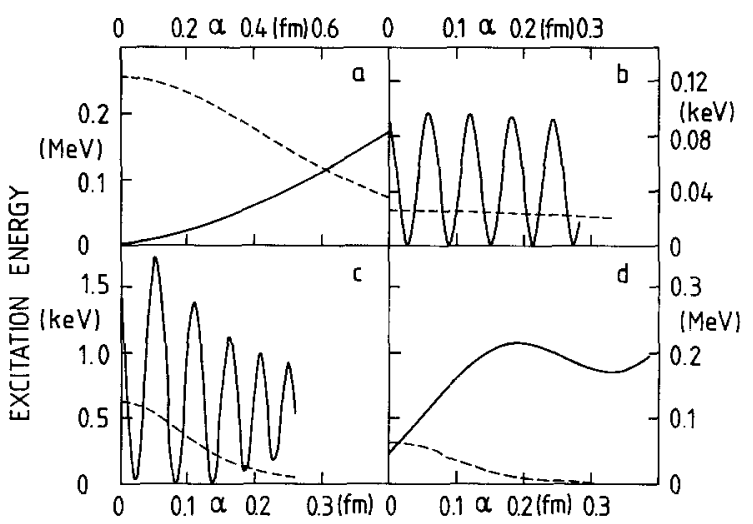

Fig. 3a-d. Plots of the exact excitation energy $E^{*}$ (solid line) and the one predicted by the cranking formula (dashed line) versus the deformation parameter $\alpha$ with fixed $\alpha_{0}=0.1 \mathrm{fm}$ and $\delta=0.5 \mathrm{MeV}$. The values of the other parameters are: $\mathbf{a} b=1, u=1 ; \mathbf{b} b=1, u$ $=0.01 ; \mathbf{c} b=5, u=0.01$ and $\mathbf{d} b=5, u=0.1$ in $\mathrm{MeV} \cdot \mathrm{fm}^{-1}$ and $\mathrm{MeV} \cdot \mathrm{fm}$ for $b$ and $u$ respectively

even if the transition probabilities are much smaller than one (typically $u=0.01-0.1 \mathrm{MeV} \mathrm{fm}$ ). On the other hand, for higher collective velocity, e.g. $u \sim 1$, the excitation energy can also be overestimated by the Inglis formula (28) (see Fig. 3a).

Obviously, from our simple two-level model one can not draw quantitative conclusions; but it is reasonable to expect that taking into account more levels will even worsen the situation as far as the validity of the cranking model is concerned, because interference effects between neighbouring crossing points can become quite important.

\section{Conclusions}

We have derived a general expression for the jumping probability between two intersecting levels; the diabatic levels were assumed to be straight lines and the collective velocity was assumed to be constant. The treatment is not restricted to the limiting case where the jumping probability is calculated between initial and final configurations which are reached for $t \rightarrow \mp \infty$ respectively, as it is the case for the Landau-Zener approximation. In our treatment the time interval during which the jumping takes place can take on any value and must not necessarily be symmetric around the time of crossing. Therefore the restrictions to straight diabatic lines and constant velocity are practically not stringent; indeed for realistic molecular or nuclear systems one always has to do with many subsequent level crossings so that a natural division into subsequent sections with rather short intervals is imposed. For these separate intervals the assumption of constant slopes and velocity are less restrictive than for the entire motion between $t=\mp \infty$. 
The jumping probability depends on the two parameters $G_{0}=\frac{b u}{2 \pi \delta^{2}}$ and $|x|=\sqrt{\frac{b}{u}}|\alpha|$ defined in (9) and (10). The LZ approximation corresponds to the limiting case $|\alpha| \rightarrow \infty$; i.e. $|x| \rightarrow \infty$ so that only the dependence on $G_{0}$ remains. For finite values of $\alpha$ the dependence of the results on $|x|$ is quite important. Moreover a discussion within the LZ-approximation where the slope $b$ and the level coupling $\delta$ is kept constant and only the velocity $u$ is varied, can be quite misleading since $|x|$ varies at the same time. A variation of $G_{0}$ while $|x|$ is kept constant means apparently a variation of the coupling $\delta$ only! An interesting peculiarity is obtained in connection with the jumping between diabatic levels near the crossing point. In this case the fact that the diabatic states are not eigenstates of the intrinsic hamiltonian imposes a time evolution which just reflects the dependence of the expansion coefficients of the diabatic states in terms of the eigenstates. Therefore, even if there is no jumping between the eigenstates, both diabatic levels will be occupied at the crossing point with equal probabilities, and with comparable probabilities in the vicinity of the crossing point.

There is an evident limitation of our treatment which is related to the two-level approximation. As we have explained above, we have to divide the process into subsequent time intervals, the lengths of which are given by the separations $\Delta \alpha$ between subsequent (avoided) crossings and the average velocity $\bar{u}$ between the crossing points, namely by $\Delta t=\frac{\Delta \alpha}{\bar{u}}$. On the other hand, by the uncertainty principle this corresponds to an energy interval $E=\frac{h}{\Delta t}=\frac{h \bar{u}}{\Delta \alpha}$ which should be small enough to contain only the pair of intersecting levels in question. If more levels are found within $\Delta E$, the two level approximation will be unreliable.

We have used our results for the transition probabilities to calculate the excitation energy within the two-level model and compared it to the cranking model. It turns out that the results of the two approaches differ in many cases even qualitatively. The results of the "exact" model depend much more sensitively on the parameters than those of the cranking model; in particular the cranking model can obviously not reproduce the oscillations which are found in the "exact" calculation. Even after averaging over the oscillations there still remain large discrepancies. This seems to be in contradiction to the generally accepted view that cranking works well for small collective velocities. However, this conclusion cannot be drawn since the limit where the collective velocity tends to zero does not exist - as is well known [10]. Apparently for the range of velocities investigated here - assumed to be typical for fission processes - the cranking results does not yet tend towards the average of the exact result.

Our present conclusions are limited by the fact that our model is very simple and does, in particular, not allow to separate collective and intrinsic motion. Nevertheless we have demonstrated that the results of an exact dynamical calculation can be extremely sensitive to the chosen boundary conditions and parameters of the model. It must be the object of further studies how this conclusion may eventually be altered by the presence of many levels and their interdependences.

\section{References}

1. Hill, D.L., Wheeler, J.A.: Phys. Rev. 89, 1102 (1953)

2. Tully, J., Preston, R.K.: J. Chem. Phys. 55, 562 (1971)

3. Nörenberg, W.: Phys. Lett. B 104, 107 (1981); Dissipative diabatic dynamics, preprint GSI-82-36, Darmstadt, GSI, 1982

4. Nörenberg, W.: Nucl. Phys. A 409, 191c (1983)

5. Landau, L.: Phys. Z. Sowjetunion 2, 46 (1932)

6. Zener, C.; Proc. R. Soc. (London) Ser. A 137, 696 (1932)

7. Crothers, D.S.F.: Adv. Mol. Phys. 17, 55 (1981)

8. Nikitin, E.E.: Comm. At. Mol. Phys. 1, 166 (1970)

9. Bárány, A., Crothers, D.S.F.: Proc. R. Soc. (London) Ser. A 385, 129 (1983)

10. Schütte, G.: Quantum mechanical description of the intrinsic excitations of fissioning nuclei. Preprint of the University of Heidelberg, FRG 1982

11. Heinrichs, J.: Phys. Rev. 176, 141 (1968)

12. Buchholz, H.: The confluent hypergeometric function. Berlin, Heidelberg, New York: Springer 1969

13. Glas, D., Mosel, U.: Phys. Lett. B 49, 301 (1974); Nucl. Phys. A 264, 268 (1976)

14. Cassing, W., Lukasiak, A., Nörenberg, W., Wang, S.J., Xu, C.O., Yadav, X.L.: Aspects of diabatic single-particle motion in heavy-ion reactions. Preprint GSI-82-13 Darmstadt, GSI, 1982

15. Abramowitz, M., Stegun, J.M.: Handbook of mathematical functions. Washington: National Bureau of Standards 1972

16. Bykhovski, V.K., Nikitin, E.E., Ovchinnikova, M.Ya.: Sov. Phys. JETP 20, 500 (1965)

17. Merzbacher, E.: Quantum mechanics. pp. 276-293. New York: John Wiley and Sons 1970

18. Thouless, D.J., Valatin, J.G.: Nucl. Phys. 31, 211 (1962)

A. Góźdź

Department of Physics

The University of Maria Sklodowska-Curie

PL-20-031 Lublin

Poland

E. Werner

M. Brack

Institut für Theoretische Physik

Universität Regensburg

Universitätsstrasse 31

D-8400 Regensburg

Federal Republic of Germany 